\title{
INFLUÊNCIA DO pH NA ETAPA SCAVENGER DE FLOTAÇÃO REVERSA DE MINÉRIO DE FERRO DA USINA DE CONCENTRAÇÃO DE BRUCUTU*
}

\author{
José Marcelino dos Reis ${ }^{1}$ \\ Aloisio Fonseca ${ }^{2}$ \\ Leandro Baptista Rodrigues ${ }^{3}$ \\ Edivânia Geralda Ribeiro ${ }^{4}$ \\ Antônio Eduardo Clark Peres ${ }^{5}$
}

Resumo

Com o empobrecimento das jazidas minerais, os novos projetos industriais e as expansões das atuais usinas de tratamento de minério de ferro, em sua grande maioria, têm sido concebidos/ adaptados para processar itabiritos pobres para produção de pellet feed. Neste contexto, a otimização dos processos já existentes e/ou a implantação de projetos mais eficazes, são medidas fundamentais para a redução do teor de ferro no rejeito; consequentemente, maximiza-se a produção e a vida útil da jazida, além de reduzir os custos operacionais. O processo de flotação reversa de minério de ferro da usina de concentração de Brucutu responde por expressiva parcela de produção, sendo constituído por quatro estágios de concentração: rougher, cleaner, recleaner e scavenger. 0 concentrado final é produzido no estágio recleaner e o rejeito final no estágio scavenger, cujo teor médio atual é de $18 \%$ Fe. Objetivando reduzir o teor de Fe no rejeito scavenger da flotação para teores próximos a $10 \%$, foi realizada uma série de testes exploratórios em bancada em diferentes faixas de $\mathrm{pH}$, com adição de soda caustica e/ou amido gelatinizado. Os resultados obtidos em escala de bancada se mostraram bastante satisfatórios apresentando considerável ganho de recuperação mássica/ metalúrgica, o que motivou a realização de um projeto para viabilizar a implementação desta proposta.

Palavras-chave: Flotação; pH; Rejeito scavenger; Recuperação.

\section{INFLUENCE OF pH ON THE SCAVENGER STAGE OF THE REVERSE FLOTATION OF IRON ORE FROM BRUCUTU'S CONCENTRATOR}

\section{Abstract}

As a consequence of the decreasing iron grades in the deposits, most recent industrial projects and expansions of the current iron ore concentrators are designed/adapted to process low grade itabirites to produce pellet feed. Under this context, the optimization of existing processes and/or the commissioning of more efficient processes are essential procedures aiming at reducing the iron content in the tailings; consequently the production and the life of the enterprise are maximized, and the OPEX is reduced. The reverse cationic iron ore flotation at Brucutu's concentrator responds for an expressive participation in the overall production, consisting of four stages: rougher, cleaner, recleaner and scavenger. The recleaner concentrate is the final concentrate and the scavenger tailings constitute the final reject product, at yearly average content of $18 \%$ Fe. Aiming at reducing the iron content in the scavenger flotation tailings to approximately $10 \%$, exploratory laboratory flotation experiments were performed at different $\mathrm{pH}$ ranges, with addition of caustic soda and/or gelatinized starch. The results achieved in the laboratory scale experiments were satisfactory, presenting considerable gains in mass and metallurgical recoveries, motivating studies addressing the possible implementation of this proposal.

Keywords: Flotation; pH; Scavenger tailings; Recovery.

1 Engenheiro de Produção, Técnico Especializado de Produção, Gerência de Tratamento de Minério, Vale, São Gonçalo do Rio Abaixo, MG, Brasil.

2 Engenheiro de Produção, Engenheiro de Processo, Gerência de Tratamento de Minério, Vale, São Gonçalo do Rio Abaixo, MG, Brasil.

3 Graduando em Engenharia de Produção, Técnico de Processo, Gerência de Tratamento de Minério, Vale, São Gonçalo do Rio Abaixo, MG, Brasil.

4 Engenheira de Produção, Técnica de Processo, Gerência de Tratamento de Minério, Vale, São Gonçalo do Rio Abaixo, MG, Brasil.

5 PhD em Engenharia Mineral, Professor Associado, Universidade Federal de Minas Gerais, Belo Horizonte, MG, Brasil.

* Contribuição técnica ao 44 Seminário de Redução de Minério de Ferro e Matérias-primas, $15^{\circ}$ Simpósio Brasileiro de Minério de Ferro e $2^{\circ}$ Simpósio Brasileiro de Aglomeração de Minério de Ferro, 15 a 18 de setembro de 2014, Belo Horizonte, MG, Brasil. 


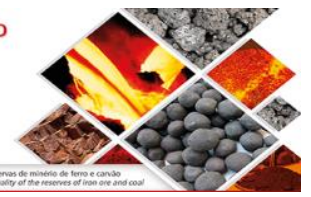

\section{INTRODUÇÃO}

Dentre os diversos processos empregados no processamento de minerais portadores de ferro pode-se dizer que, atualmente, a flotação é o principal método utilizado para concentração de superfinos. Sua importância se deve à comprovada seletividade para minérios de ferro, à ampla faixa de tamanho e teores em que ela se aplica e às altas taxas de alimentação permitidas.

Como a flotação é um processo que explora diferenças nas características de superfície entre as várias espécies minerais presentes numa fase aquosa, é fundamental conhecer quais as propriedades de superfície capazes de afetar a sua dinâmica, bem como a atuação e seletividade dos reagentes de flotação $[1,2]$.

A adição criteriosa de determinados reagentes possibilita à flotação transformar, seletivamente, a superfície de partículas minerais hidrofílicas em hidrofóbicas. A probabilidade de êxito deste processo será tanto maior quanto mais distintas forem as superfícies das espécies a serem separadas.

Atualmente, todas as grandes usinas brasileiras produtoras de pellet feed utilizam a amina como coletor/espumante e amido como depressor no processo de flotação reversa de minério de ferro. Grandes empresas do ramo químico vêm desenvolvendo e estudando depressores sintéticos para esta aplicação. Como depressor de minerais de ferro, a adição do amido é realizada previamente a adição do coletor, sendo um dos reagentes que mais influenciam na recuperação metalúrgica do processo [3].

As grandes perdas relacionadas ao processo de flotação estão ligadas à indesejável perda de partículas finas para rejeito. Na flotação, assim como em outros processos que envolvem a partição de fluxos de polpa, partículas menores que um determinado tamanho crítico acompanham a partição de água, o que, consequentemente, ocasiona perda de seletividade do processo de concentração.

Leja [4] acredita que partículas minerais finas apresentem menor intensidade de adsorção de amido que partículas minerais grossas. Consequentemente, haveria um maior arraste de partículas minerais de ferro para o flotado, resultando na indesejável diminuição tanto de recuperação metalúrgica quanto da área superficial específica do concentrado. Tal suspeita se basearia no fato de que partículas com maior área superficial exibiriam maior número de sítios para ancoramento da molécula do reagente, tornando a adsorção mais intensa. Entretanto, essa observação é questionável se considerarmos que, por menor que seja a área superficial de uma partícula numa flotação industrial, ainda assim, será muito maior que a molécula do depressor [4].

Leja [4] afirma também que a ação floculante do amido é fundamental para a recuperação de partículas de menor granulometria no afundado, através da formação de flocos que são mais facilmente deprimidos. Neste contexto, deve-se destacar que o condicionamento da polpa mineral com amido é feito numa condição favorável para a estabilidade do sistema, pois o amido, gelatinizado com soda, aumenta drasticamente o pH da polpa, elevando a dispersão do sistema. Quanto menor a partícula, maior será esse efeito e, desse modo, mais difícil a recuperação delas no afundado. A escolha de um depressor não deve ser baseada somente no seu desempenho, mas também por fatores externos como preço, disponibilidade, biodegradabilidade, toxicidade, além das variáveis de processo, como características do minério e do coletor, entre outras [4].

A amina se constitui como um dos principais grupos de coletores catiônicos e sua adsorção sobre a superfície do quartzo, sob a forma iônica, em meio aquoso, se dá

* Contribuição técnica ao 44 Seminário de Redução de Minério de Ferro e Matérias-primas, $15^{\circ}$ Simpósio Brasileiro de Minério de Ferro e 2o Simpósio Brasileiro de Aglomeração de Minério de Ferro, 15 a 18 de setembro de 2014, Belo Horizonte, MG, Brasil. 
por um mecanismo de atração eletrostática, já que a superfície do mineral está carregada negativamente em valores de $\mathrm{pH}$ superiores a 2,5.

Aminas estão totalmente dissociadas desde a faixa ácida até valores levemente alcalinos de $\mathrm{pH}$. Amina molecular é formada em $\mathrm{pH}$ alcalino, sendo que na faixa de $\mathrm{pH}$ entre 8,5 e 11 a amina encontra-se tanto na forma molecular quanto iônica.

$\mathrm{O} \mathrm{pH}$ constitui-se como uma das principais variáveis dos sistemas de flotação de minerais oxidados de ferro e quartzo, tanto por governar a dissociação e hidrólise dos reagentes coletores, quanto pela dependência das cargas de superfície das partículas destes minerais às concentrações dos íons $\mathrm{H}^{+}$e $\mathrm{OH}^{-}$, em solução. Para a flotação de minério de ferro, considera-se que a faixa ideal de $\mathrm{pH}$ se mantenha entre 10.0 e 10.5, pois, é nesta faixa que a amina se encontra 50\% dissociada, apresentando-se com função coletora e espumante ao mesmo tempo. O principal regulador de $\mathrm{pH}$ utilizado industrialmente é a soda caustica $(\mathrm{NaOH})$, que tem a função de elevar o pH para os patamares adequados.

O atual processo de flotação da Usina de Brucutu é constituído por quatro estágios de concentração: rougher, cleaner, recleaner e scavenger. $O$ concentrado final do circuito é produzido no estágio recleaner e o rejeito final no estágio scavenger. No layout atual, o concentrado do circuito de flotação scavenger tem as seguintes opções:

a) Alimentar o peneiramento de alta frequência para retirada de quartzo grosso da carga circulante do processo, e/ou

b) Alimentar dois concentradores magnéticos tipo carrossel para produção de pellet feed.

O circuito de flotação da usina de Brucutu gera atualmente rejeito final com teor de Fe da ordem de $18 \%$ e o pH usual é de 9.8 , sendo um dos fatores determinantes para a obtenção de qualidade no produto final dado ao elevado percentual acima de $0,15 \mathrm{~mm}$ na alimentação desde circuito.

Caso se viabilize a implementação desta melhoria no processo, a proposta inclui a incorporação do undersize do peneiramento de alta frequência ao fluxo proveniente da segunda deslamagem, juntamente com o produto da moagem após deslamagem e assim alimentar o "circuito de finos". Este circuito está em fase de montagem e será composto por cinco tanques células, resultante da expansão de Brucutu. Esta otimizaçao por si só não garante a redução do teor de ferro do rejeito scavenger para valores da ordem de $10 \%$, sendo necessário também melhorar as condições operacionais e de processo deste circuito. A figura 1 a seguir apresenta um fluxograma simplificado desse processo de flotação, considerando a inclusão supracitada.

Os atuais níveis de consumos de amido e amina na flotação convencional são da ordem de $600 \mathrm{~g} / \mathrm{t}$ de alimentação nova e $300 \mathrm{~g} / \mathrm{t}$ de $\mathrm{SiO}_{2}$, respectivamente. 


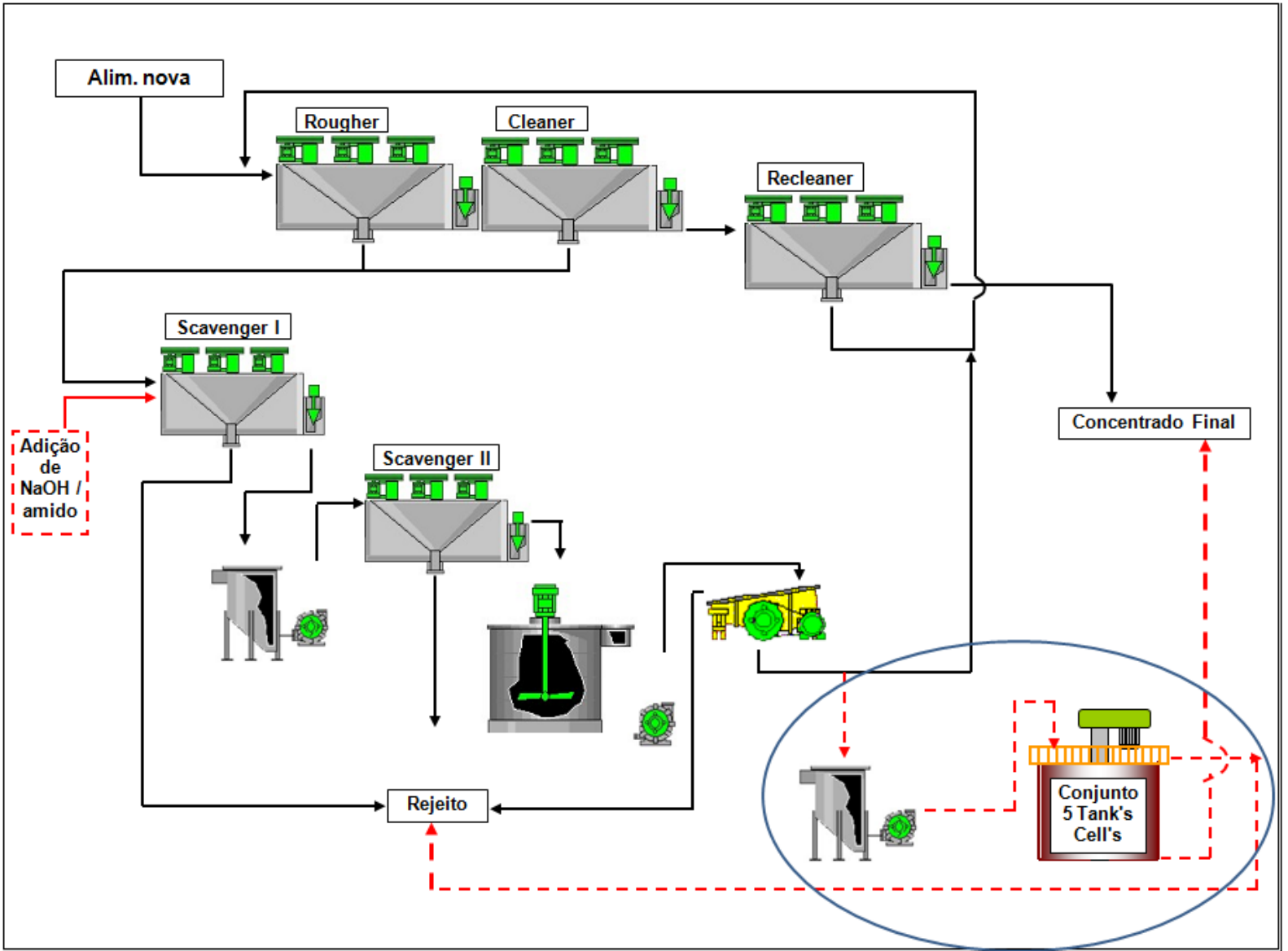

Figura 1 - Fluxograma simplificado do circuito de flotação

\section{OBJETIVO}

Adequar o $\mathrm{pH}$ do circuito scavenger de flotação com o intuito de reduzir o teor de $\mathrm{Fe}$ no rejeito final da flotação reversa de minério de $\mathrm{Fe}$ da Usina de Brucutu de $18 \%$ para teores próximos de $10 \%$, o que representaria um aumento de produção na usina de concentração entre $4,5 \%$ e 5,5\%.

\section{MATERIAIS E MÉTODOS}

As amostras para realização dos testes de bancada foram coletadas nas quatro linhas de alimentação da flotação scavenger, de modo a gerar uma amostra representativa do processo. Posteriormente, as amostras foram encaminhadas ao laboratório de processo para preparação e realização dos testes. A etapa de preparação consistiu em quartear a amostra de polpa em quarteador de polpa para retiradas das alíquotas para análises físico-químicas e testes de bancada.

A $1^{\text {a }}$ etapa de testes de flotação em bancada foi realizada de modo comparativo, visando avaliar qual seria a faixa de $\mathrm{pH}$ mais adequada para obtenção do teor de ferro desejado no rejeito final $(\mathrm{Fe}<\mathrm{ou}=10 \%)$ comparada a condição de $\mathrm{pH}$ atual $(\mathrm{pH}<\mathrm{ou}=9,0)$.

A $2^{\text {a }}$ etapa de testes consistiu em submeter as amostras de alimentação do scavenger a cinéticas de flotação em três diferentes condições. Os produtos gerados nas cinéticas foram submetidos a distribuição granuloquímica para verificar a distribuição dos teores de ferro e sílica. 


\section{RESULTADOS E DISCUSSÃO}

Tabela 1 - Resumo dos resultados dos testes de bancada - 1a etapa

\begin{tabular}{|c|c|c|c|c|c|c|c|c|c|c|}
\hline \multirow{2}{*}{ Teste } & \multirow{2}{*}{$\begin{array}{l}\text { Condiçöes do } \\
\text { teste }\end{array}$} & \multirow{2}{*}{ pH } & \multicolumn{2}{|c|}{ Recuperação (\%) } & \multicolumn{2}{|c|}{$\begin{array}{c}\text { Alimentação } \\
\text { calculada }\end{array}$} & \multicolumn{2}{|c|}{ Concentrado } & \multicolumn{2}{|c|}{ Rejeito } \\
\hline & & & Mássica & Metálica & $\mathrm{Fe}$ & $\mathrm{SiO} 2$ & $\mathrm{Fe}$ & $\mathrm{SiO} 2$ & $\mathrm{Fe}$ & $\mathrm{SiO} 2$ \\
\hline 1 & $\begin{array}{c}\text { Polpa natural (40\% } \\
\text { Solidos) }\end{array}$ & 9,05 & 55,4 & 86,0 & 36,28 & 45,99 & 56,31 & 18,30 & 11,41 & 80,38 \\
\hline 2 & $\begin{array}{l}\text { Adição de } \mathrm{NaOH} \\
\quad(70 \mathrm{~g} / t)\end{array}$ & 9,54 & 55,9 & 87,3 & 36,19 & 46,55 & 56,55 & 18,45 & 10,44 & 82,09 \\
\hline 3 & $\begin{array}{c}\text { Adição de } \mathrm{NaOH} \\
(110 \mathrm{~g} / \mathrm{t})\end{array}$ & 10,00 & 56,7 & 87,6 & 34,66 & 48,57 & 53,52 & 21,90 & 9,95 & 83,50 \\
\hline 4 & $\begin{array}{l}\text { Adição de } \mathrm{NaOH} \\
(150 \mathrm{~g} / \mathrm{t})\end{array}$ & 10,63 & 58,0 & 88,5 & 34,72 & 48,89 & 53,00 & 23,38 & 9,49 & 84,10 \\
\hline 5 & $\begin{array}{l}\text { Adição } 600 \mathrm{~g} / \mathrm{t} \\
\text { amido }\end{array}$ & 9,61 & 56,9 & 87,8 & 34,68 & 48,68 & 53,50 & 21,95 & 9,81 & 84,00 \\
\hline 6 & $\begin{array}{l}\text { Adição } 1000 \mathrm{~g} / \mathrm{t} \\
\text { amido }\end{array}$ & 10,02 & 58,8 & 89,5 & 34,67 & 48,75 & 52,82 & 23,22 & 8,79 & 85,16 \\
\hline 7 & $\begin{array}{l}\text { Adição } 1500 \mathrm{~g} / \mathrm{t} \\
\text { amido }\end{array}$ & 10,56 & 64,7 & 91,2 & 34,96 & 48,78 & 49,26 & 28,84 & 8,74 & 85,32 \\
\hline
\end{tabular}

A amostra de alimentação da flotação scavenger testada em bancada continha residual de amido proveniente do processo industrial, cuja dosagem foi de $600 \mathrm{~g} / \mathrm{t}$. $\mathrm{Na}$ tabela 1 se observa que os testes 2 a 4 realizados com adição gradativa de soda, comparados à condição natural (teste 1), apresentaram ganhos de recuperação em função da redução proporcional do teor de ferro no rejeito.

Para os testes 5 a 7 realizados com a mesma amostra, onde se fez dosagens adicionais de amido (600 a 1500g/t) observa que os teores de ferro no rejeito se mantiveram em patamares próximos a condição apresentada nos testes realizados com soda, mesmo para a dosagem de mais $1500 \mathrm{~g} / \mathrm{t}$.

Para ambas as condições de testes, comparadas a natural, percebe-se que há uma tendência de elevação do teor de $\mathrm{SiO}_{2}$ no concentrado, ocasionado pelo menor poder de coleta da amina e pela elevação do $\mathrm{pH}$.

Os resultados obtidos na $11^{\underline{a}}$ etapa dos testes de bancada mostraram que, a partir da elevação do pH com adição de soda caustica, é possível reduzir o teor de Fe no rejeito scavenger da flotação para valores inferiores a $10 \%$. Testes realizados com dosagens adicionais de amido não apresentaram desempenho muito superior aos testes realizados com adição de soda; portanto, não se justificaria agregar esse custo adicional ao processo.

Visando um melhor entendimento do comportamento da amostra durante o processo de flotação, foram realizadas três cinéticas de flotação em três diferentes faixas de $\mathrm{pH}$. Os concentrados e rejeitos gerados foram submetidos a distribuição granuloquímica.

* Contribuição técnica ao 44 Seminário de Redução de Minério de Ferro e Matérias-primas, 15ํ Simpósio Brasileiro de Minério de Ferro e $2^{\circ}$ Simpósio Brasileiro de Aglomeração de Minério de Ferro, 15 a 18 de setembro de 2014, Belo Horizonte, MG, Brasil. 
Tabela 2 - Resultado da cinética de flotação 1 - Condição natural $(\mathrm{pH} 9,05)$

\begin{tabular}{c|c|c|c|c|c|c|c|c}
\multirow{2}{*}{ Produtos } & \multicolumn{3}{|c|}{ Recuperação (\%) } & \multicolumn{5}{c}{ Teores (\%) } \\
\cline { 2 - 9 } & Mássica & $\mathbf{F e}$ & $\mathbf{S i O 2}$ & $\mathbf{F e}$ & $\mathbf{S i O 2}$ & $\mathbf{P}$ & $\mathbf{A l}_{2} \mathrm{O}_{3}$ & Fech. Químico \\
\hline Alim. Calc. & 100,0 & - & - & 25,01 & 61,44 & 0,027 & 1,13 & 98,65 \\
\hline Concentrado & $\mathbf{2 2 , 7}$ & $\mathbf{6 0 , 2}$ & $\mathbf{0 , 9}$ & $\mathbf{6 6 , 3 1}$ & $\mathbf{2 , 4 9}$ & 0,040 & 0,82 & 98,56 \\
\hline Rejeito Global & $\mathbf{7 7 , 3}$ & $\mathbf{3 9 , 8}$ & $\mathbf{9 9 , 1}$ & $\mathbf{1 2 , 8 7}$ & 78,77 & 0,024 & 1,22 & 98,68 \\
\hline Rejeito 1 & 27,6 & - & - & 11,99 & 80,29 & 0,023 & 1,28 & 99,01 \\
\hline Rejeito 2 & 26,5 & - & - & 10,40 & 83,00 & 0,018 & 0,95 & 99,06 \\
\hline Rejeito 3 & 17,8 & - & - & 14,16 & 76,29 & 0,025 & 1,27 & 98,10 \\
\hline Rejeito 4 & 5,4 & - & - & 25,18 & 58,52 & 0,049 & 2,02 & 97,04 \\
\hline
\end{tabular}

Tabela 3 - Resultado da cinética de flotação 2 - Adição de NaOH 150 g/t (pH 10,20)

\begin{tabular}{c|c|c|c|c|c|c|c|c}
\multirow{2}{*}{ Produtos } & \multicolumn{3}{|c|}{ Recuperação (\%) } & \multicolumn{5}{c}{ Teores (\%) } \\
\cline { 2 - 9 } & Mássica & $\mathrm{Fe}$ & $\mathrm{SiO2}$ & $\mathrm{Fe}$ & $\mathrm{SiO} 2$ & $\mathrm{P}$ & $\mathrm{Al}_{2} \mathrm{O}_{3}$ & Fech. Químico \\
\hline Alim. Calc. & 100,0 & - & - & 24,01 & 63,09 & 0,025 & 1,04 & 98,77 \\
\hline Concentrado & $\mathbf{2 3 , 0}$ & $\mathbf{6 1 , 8}$ & $\mathbf{1 , 8}$ & $\mathbf{6 4 , 5 3}$ & $\mathbf{4 , 8 3}$ & 0,041 & 0,86 & 98,38 \\
\hline Rejeito Global & $\mathbf{7 7 , 0}$ & $\mathbf{3 8 , 2}$ & $\mathbf{9 8 , 2}$ & $\mathbf{1 1 , 9 2}$ & 80,48 & 0,020 & 1,10 & 98,88 \\
\hline Rejeito 1 & 22,1 & - & - & 9,36 & 84,32 & 0,018 & 1,04 & 98,99 \\
\hline Rejeito 2 & 21,5 & - & - & 9,28 & 84,63 & 0,016 & 0,89 & 99,01 \\
\hline Rejeito 3 & 20,0 & - & - & 13,94 & 77,56 & 0,022 & 1,17 & 98,93 \\
\hline
\end{tabular}

Tabela 4 - Resultado da cinética de flotação 3 - adição de amido 600 g/t (pH 10,69)

\begin{tabular}{c|c|c|c|c|c|c|c|c}
\multirow{2}{*}{ Produtos } & \multicolumn{3}{|c|}{ Recuperação (\%) } & \multicolumn{5}{c}{ Teores (\%) } \\
\cline { 2 - 9 } & Mássica & $\mathrm{Fe}$ & $\mathbf{S i O 2}$ & $\mathbf{F e}$ & $\mathbf{S i O 2}$ & $\mathbf{P}$ & $\mathrm{Al}_{2} \mathrm{O}_{3}$ & Fech. Quimico \\
\hline Alim. Calc. & 100,0 & - & - & 26,53 & 58,17 & 0,028 & 1,10 & 97,53 \\
\hline Concentrado & $\mathbf{3 1 , 2}$ & $\mathbf{7 4 , 8}$ & $\mathbf{3 , 0}$ & $\mathbf{6 3 , 6 5}$ & $\mathbf{5 , 6 0}$ & 0,045 & 0,89 & 97,94 \\
\hline Rejeito Global & $\mathbf{6 8 , 8}$ & $\mathbf{2 5 , 2}$ & $\mathbf{9 7 , 0}$ & $\mathbf{9 , 7 3}$ & 81,95 & 0,020 & 1,20 & 97,35 \\
\hline Rejeito 1 & 20,3 & - & - & 12,74 & 77,25 & 0,025 & 1,49 & 97,62 \\
\hline Rejeito 2 & 16,2 & - & - & 8,11 & 84,75 & 0,018 & 1,05 & 97,13 \\
\hline Rejeito 3 & 19,8 & - & - & 7,70 & 84,87 & 0,017 & 1,01 & 97,32 \\
\hline Rejeito 4 & 12,5 & - & - & 10,16 & 81,35 & 0,020 & 1,20 & 97,35 \\
\hline
\end{tabular}

Índice de seletividade Gaudin ===>

9,78

As cinéticas confirmaram a tendência de redução do teor de Fe no rejeito scavenger da flotação ao se elevar o pH da polpa para valores acima de 10. Comparando os resultados dos testes entre si, observa-se que ao se fazer a adição de soda caustica, a redução do teor de ferro no rejeito é discreta, assim como a recuperação

* Contribuição técnica ao 44 Seminário de Redução de Minério de Ferro e Matérias-primas, 15o Simpósio Brasileiro de Minério de Ferro e 2o Simpósio Brasileiro de Aglomeração de Minério de Ferro, 15 a 18 de setembro de 2014, Belo Horizonte, MG, Brasil. 
de ferro em relação a condição natural. Com a dosagem adicional de amido observa-se que ocorre um significativo aumento na recuperação mássica e metálica, no entanto, ocorre também maior retenção de sílica no concentrado. Uma análise preliminar dos ganhos de recuperação obtidos com a adição de amido justificaria o custo-benefício, dobrando o consumo atual (600 g/t). Embora nos testes de bancada esta redução seja de 3 pontos percentuais, operacionalmente, este ganho pode ser bastante significativo comparados aos atuais teores de ferro praticados no rejeito da flotação scavenger, da ordem de $19 \%$.

As tabelas 5, 6 e 7 apresentam as análises granuloquímicas dos concentrados das cinéticas de flotação.

Tabela 5 - Granuloquímica do concentrado - Cinética 1 ( $\mathrm{pH}$ natural 9,05)

\begin{tabular}{c|c|c|c|c|c|c|c|c}
\multirow{2}{*}{ Fração } & \multicolumn{3}{|c|}{ Recuperação (\%) } & \multicolumn{5}{c}{ Teores (\%) } \\
\cline { 2 - 10 } & Massa & $\mathbf{F e}$ & $\mathbf{S i O}_{2}$ & $\mathbf{F e}$ & $\mathbf{S i O}_{2}$ & $\mathbf{P}$ & $\mathbf{A l}_{2} \mathbf{O}_{\mathbf{3}}$ & Fech. Quimico \\
\hline$+0,15 \mathrm{~mm}$ & 6,6 & 4,2 & 66,3 & 41,67 & 31,92 & 0,114 & 3,56 & 100,83 \\
\hline$-0,15 \mathrm{~mm}$ & 93,4 & 95,8 & 33,7 & 67,81 & 1,15 & 0,039 & 0,80 & 100,20 \\
\hline Global & $\mathbf{1 0 0}$ & 100 & 100 & $\mathbf{6 6 , 0 8}$ & $\mathbf{3 , 1 9}$ & $\mathbf{0 , 0 4 4}$ & $\mathbf{0 , 9 8}$ & $\mathbf{1 0 0 , 2 4}$ \\
\hline
\end{tabular}

Tabela 6 - Granuloquímica do concentrado - Cinética 2 (pH 10,20)

\begin{tabular}{c|c|c|c|c|c|c|c|c}
\multirow{2}{*}{ Fraçăo } & \multicolumn{3}{|c|}{ Recuperaçäo (\%) } & \multicolumn{5}{c}{ Teores (\%) } \\
\cline { 2 - 10 } & Massa & $\mathbf{F e}$ & $\mathbf{S i O}_{2}$ & $\mathbf{F e}$ & $\mathbf{S i O}_{2}$ & $\mathbf{P}$ & $\mathbf{A l}_{2} \mathrm{O}_{3}$ & Fech. Quimico \\
\hline$+0,15 \mathrm{~mm}$ & 8,3 & 4,0 & 76,7 & 30,94 & 51,90 & 0,036 & 1,10 & 99,26 \\
\hline$-0,15 \mathrm{~mm}$ & 91,7 & 96,0 & 23,3 & 67,47 & 1,43 & 0,046 & 0,95 & 99,33 \\
\hline Global & $\mathbf{1 0 0}$ & 100 & 100 & $\mathbf{6 4 , 4 4}$ & $\mathbf{5 , 6 2}$ & $\mathbf{0 , 0 4 5}$ & $\mathbf{0 , 9 6}$ & $\mathbf{9 9 , 3 3}$ \\
\hline
\end{tabular}

Tabela 7 - Granuloquímica do concentrado - Cinética $3(\mathrm{pH} 10,69)$

\begin{tabular}{c|c|c|c|c|c|c|c|c}
\multirow{2}{*}{ Fração } & \multicolumn{3}{|c|}{ Recuperação $(\%)$} & \multicolumn{5}{c}{ Teores $(\%)$} \\
\cline { 2 - 10 } & Massa & $\mathbf{F e}$ & $\mathbf{S i O}_{2}$ & $\mathbf{F e}$ & $\mathbf{S i O}_{2}$ & $\mathbf{P}$ & $\mathbf{A l}_{2} \mathrm{O}_{3}$ & Fech. Químico \\
\hline$+0,15 \mathrm{~mm}$ & 11,0 & 4,6 & 84,6 & 26,48 & 55,39 & 0,073 & 2,49 & 99,55 \\
\hline$-0,15 \mathrm{~mm}$ & 89,0 & 95,4 & 15,4 & 67,72 & 1,25 & 0,047 & 0,87 & 99,42 \\
\hline Global & $\mathbf{1 0 0}$ & 100 & 100 & $\mathbf{6 3 , 1 8}$ & $\mathbf{7 , 2 1}$ & $\mathbf{0 , 0 5 0}$ & $\mathbf{1 , 0 5}$ & $\mathbf{9 9 , 4 3}$ \\
\hline
\end{tabular}

As tabelas 5, 6 e 7 mostram que houve um engrossamento dos concentrados de flotação com a elevação do $\mathrm{pH}$, resultando num aumento gradativo de massa na fração $+0,15 \mathrm{~mm}$. Com relação aos valores de recuperação metálica, nota-se que o ferro se concentrou, predominantemente, na fração $-0,15 \mathrm{~mm}$ (acima de $95 \%$ ); enquanto a sílica se concentrou na fração no oversize. Houve um ligeiro desvio nos teores recalculados em relação aos obtidos nos concentrados da cinética de flotação.

Os resultados mostram também que o incremento gradativo de massa na fração + $0,15 \mathrm{~mm}$ está relacionado ao menor poder de coleta da amina, provocado pela elevação do $\mathrm{pH}$, resultando em maior presença de sílica grosseira no concentrado. Tal situação ratifica a necessidade de manutenção do circuito de peneiramento de alta frequência para retirada de sílica grosseira do concentrado scavenger da flotação, sendo o fluxo passante incorporado à alimentação dos tanques células após condicionamento com amido.

$\mathrm{Na}$ tabela 8, apresenta-se um comparativo de recuperação de ferro e qualidade dos produtos obtidos nas etapas de flotação e peneiramento em $0,15 \mathrm{~mm}$.

* Contribuição técnica ao 44 Seminário de Redução de Minério de Ferro e Matérias-primas, 15ํ Simpósio Brasileiro de Minério de Ferro e $2^{\circ}$ Simpósio Brasileiro de Aglomeração de Minério de Ferro, 15 a 18 de setembro de 2014, Belo Horizonte, MG, Brasil. 
Tabela 8 - Comparativo de recuperação de ferro e qualidade por etapa, em função da adição de reagentes.

\begin{tabular}{|c|c|c|c|c|c|c|c|}
\hline \multirow{2}{*}{ Etapa } & \multirow{2}{*}{\begin{tabular}{|c|} 
Parâmetros \\
Elementos analisados $\Rightarrow$
\end{tabular}} & \multicolumn{2}{|c|}{ CINÉTICA 1 - pH: 9,05} & \multicolumn{2}{|c|}{ CINÉTICA 2 - pH: 10,20 } & \multicolumn{2}{|c|}{ CINÉTICA 3 - pH: 10,69 } \\
\hline & & $\mathrm{Fe}(\%)$ & $\mathrm{SiO} 2(\%)$ & $\mathrm{Fe}(\%)$ & $\mathrm{SiO} 2(\%)$ & $\mathrm{Fe}(\%)$ & $\mathrm{SiO} 2(\%)$ \\
\hline \multirow{4}{*}{ Flotaçäo } & Teor Concent. Flotação & 66,31 & 2,49 & 64,53 & 4,83 & 63,65 & 5,60 \\
\hline & Recup. Mássica Fe (\%) & \multicolumn{2}{|c|}{22,7} & \multicolumn{2}{|c|}{23,0} & \multicolumn{2}{|c|}{31,2} \\
\hline & Recup. Metálica Fe (\%) & \multicolumn{2}{|c|}{60,2} & \multicolumn{2}{|c|}{61,8} & \multicolumn{2}{|c|}{74,8} \\
\hline & Adição de reagentes & \multicolumn{2}{|c|}{ condição natural } & \multicolumn{2}{|c|}{$\mathrm{NaOH}-150 \mathrm{~g} / \mathrm{t}$} & \multicolumn{2}{|c|}{ Amido - $600 \mathrm{~g} / \mathrm{t}$} \\
\hline \multirow{4}{*}{$\begin{array}{l}\text { Peneiramento } \\
\text { Concentrado } \\
\text { de Flotaçäo }\end{array}$} & Elementos analisados $\Longrightarrow$ & $\mathrm{Fe}(\%)$ & $\mathrm{SiO} 2(\%)$ & $\mathrm{Fe}(\%)$ & $\mathrm{SiO} 2(\%)$ & $\mathrm{Fe}(\%)$ & $\mathrm{SiO} 2(\%)$ \\
\hline & Teor Fração < 0,15mm (\%) & 67,81 & 1,15 & 67,47 & 1,43 & 67,72 & 1,25 \\
\hline & Recup. Mássica Fe (\%) & \multicolumn{2}{|c|}{93,4} & \multicolumn{2}{|c|}{91,7} & \multicolumn{2}{|c|}{89,0} \\
\hline & Recup. Metálica Fe (\%) & \multicolumn{2}{|c|}{95,8} & \multicolumn{2}{|c|}{96,0} & \multicolumn{2}{|c|}{95,4} \\
\hline
\end{tabular}

Embasado na tabela 8 onde são apresentados os resultados comparativos entre as etapas de flotação e peneiramento (fração $<0,15 \mathrm{~mm}$ ), pode-se fazer as seguintes considerações:

- Qualidade dos produtos: observa-se que houve uma redução significativa do teor de $\mathrm{SiO}_{2}$ da fração $<0,15 \mathrm{~mm}$ do peneiramento em relação aos teores obtidos nos concentrados da cinética de flotação, se mantendo ligeiramente acima de $1 \%$;

- Recuperação em massa de Fe: enquanto a flotação apresentou ganho crescente de massa na medida em que se elevou o pH da polpa, no peneiramento a situação se inverteu, houve um decréscimo proporcional para a mesma condição. Este fato ratifica que a elevação do $\mathrm{pH}$ proporciona aumento de massa no concentrado de flotação; entretanto, contribui também para a presença de sílica grosseira (> $0,15 \mathrm{~mm})$.

- Recuperação metálica de Fe: observa-se que na flotação a recuperação metálica de ferro tende a acompanhar a recuperação em massa, isto é, crescente com a elevação do $\mathrm{pH}$. No peneiramento, nota-se que, independentemente da faixa de $\mathrm{pH}$ testada, a massa metálica tende a se concentrar nesta fração $(<0,15 \mathrm{~mm})$, superior a $95 \%$.

As tabelas 9, 10 e 11 apresentam as análises granuloquímicas dos rejeitos das cinéticas de flotação.

Tabela 9 - Granuloquímica do rejeito - Cinética $1(\mathrm{pH}$ natural 9,05)

\begin{tabular}{c|c|c|c|c|c|c|c|c}
\multirow{2}{*}{ Fração } & \multicolumn{4}{|c|}{ Recuperaçăo (\%) } & \multicolumn{5}{c}{ Teores (\%) } \\
\cline { 2 - 9 } & Massa & $\mathbf{F e}$ & $\mathbf{S i O}_{2}$ & $\mathbf{F e}$ & $\mathbf{S i O}_{2}$ & $\mathbf{P}$ & $\mathbf{A l}_{2} \mathbf{O}_{3}$ & Fech. Químico \\
\hline$+0,044 \mathrm{~mm}$ & 81,9 & 24,6 & 95,3 & 3,58 & 94,13 & 0,010 & 0,49 & 100,2 \\
\hline$-0,044 \mathrm{~mm}$ & 18,1 & 75,4 & 4,7 & 49,75 & 21,17 & 0,090 & 3,28 & 100,2 \\
\hline Global & $\mathbf{1 0 0}$ & 100 & 100 & $\mathbf{1 1 , 9 4}$ & $\mathbf{8 0 , 9 2}$ & $\mathbf{0 , 0 2 4}$ & $\mathbf{0 , 9 9}$ & $\mathbf{1 0 0 , 2}$ \\
\hline
\end{tabular}

Tabela 10 - Granuloquímica do rejeito - Cinética $2(\mathrm{pH} 10,20)$

\begin{tabular}{c|c|c|c|c|c|c|c|c}
\multirow{2}{*}{ Fração } & \multicolumn{4}{|c|}{ Recuperação (\%) } & \multicolumn{5}{c}{ Teores (\%) } \\
\cline { 2 - 9 } & Massa & $\mathbf{F e}$ & $\mathbf{S i O}_{2}$ & $\mathbf{F e}$ & $\mathbf{S i O}_{2}$ & $\mathbf{P}$ & $\mathbf{A l}_{2} \mathbf{O}_{3}$ & Fech. Quimico \\
\hline$+0,044 \mathrm{~mm}$ & 82,8 & 27,2 & 95,9 & 3,95 & 92,64 & 0,010 & 0,42 & 99,1 \\
\hline$-0,044 \mathrm{~mm}$ & 17,2 & 72,8 & 4,1 & 51,10 & 19,13 & 0,088 & 3,16 & 100,0 \\
\hline Global & $\mathbf{1 0 0}$ & 100 & 100 & $\mathbf{1 2 , 0 4}$ & $\mathbf{8 0 , 0 3}$ & $\mathbf{0 , 0 2 3}$ & $\mathbf{0 , 8 9}$ & $\mathbf{9 9 , 2}$ \\
\hline
\end{tabular}

* Contribuição técnica ao 44 Seminário de Redução de Minério de Ferro e Matérias-primas, $15^{\circ}$ Simpósio Brasileiro de Minério de Ferro e 2o Simpósio Brasileiro de Aglomeração de Minério de Ferro, 15 a 18 de setembro de 2014, Belo Horizonte, MG, Brasil. 
Tabela 11 - Granuloquímica do rejeito - Cinética 3(pH 10,69)

\begin{tabular}{c|c|c|c|c|c|c|c|c}
\multirow{2}{*}{ Fração } & \multicolumn{4}{|c|}{ Recuperação (\%) } & \multicolumn{5}{c}{ Teores (\%) } \\
\cline { 2 - 9 } & Massa & $\mathbf{F e}$ & $\mathbf{S i O}_{2}$ & $\mathbf{F e}$ & $\mathbf{S i O}_{2}$ & $\mathbf{P}$ & $\mathbf{A l}_{2} \mathbf{O}_{3}$ & Fech. Químico \\
\hline$+0,044 \mathrm{~mm}$ & 86,4 & 31,2 & 96,1 & 3,31 & 94,64 & 0,010 & 0,60 & 100,4 \\
\hline$-0,044 \mathrm{~mm}$ & 13,6 & 68,8 & 3,9 & 46,48 & 24,68 & 0,091 & 3,73 & 99,8 \\
\hline Global & $\mathbf{1 0 0}$ & 100 & 100 & $\mathbf{9 , 1 6}$ & $\mathbf{8 5 , 1 5}$ & $\mathbf{0 , 0 2 1}$ & $\mathbf{1 , 0 2}$ & $\mathbf{1 0 0 , 4}$ \\
\hline
\end{tabular}

Com base nos resultados apresentados nas tabelas 9, 10 e 11, podem ser feitas as seguintes considerações:

- Fisicamente, as amostras de rejeito analisadas se apresentam com mais de $80 \%$ de suas massas na fração $+0,044 \mathrm{~mm}$. Embora a fração $-0,044 \mathrm{~mm}$ se apresente com menor percentual de massa, observa-se que 0 ferro está contido predominantemente nesta (cerca de $70 \%$ );

- Quimicamente, a fração $+0,044 \mathrm{~mm}$ se apresenta extremamente pobre em ferro, sendo que a recuperação de $\mathrm{SiO}_{2}$ nesta fração é superior a 95\%. Também se observa-se um ligeiro desvio nos teores recalculados em relação aos obtidos nos rejeitos da cinética de flotação.

As análises granuloquímicas mostraram que o ferro presente nas amostras de rejeito se apresenta predominantemente na fração fina $(-0,044 \mathrm{~mm})$, o que dificulta bastante a sua recuperação em célula de flotação convencional.

A tabela 12 apresentada a seguir, mostra que tecnicamente é viável a implementação desta melhoria pela expectativa de consideráveis ganhos de produção para a usina de concentração de Brucutu.

Tabela 12 - Análise de Viabilidade Técnica

\begin{tabular}{|c|c|c|c|}
\hline \multicolumn{4}{|c|}{ Análise de Viabilidade Técnica - Adequação do pH Scavenger da Flotação } \\
\hline Premissas & Tal Qual & Soda $=150 \mathrm{~g} / \mathrm{t}$ & Amido $=600 \mathrm{~g} / \mathrm{t}$ \\
\hline Alimentação Nova Flotação (t) & 24.672 .504 & 24.672 .504 & 24.672 .504 \\
\hline Consumo Soda (t) & - & 3.701 & 2.467 \\
\hline Consumo Amido (t) & - & - & 14.804 \\
\hline Custo adicional de reagentes (R\$) & - & $\mathrm{R} \$ \quad 14.803 .502,65$ & $\mathrm{R} \$ \quad 21.711 .803,89$ \\
\hline Teor Fe Alimentação (\%) & 50,97 & 50,97 & 50,97 \\
\hline Teor Fe Concentrado (\%) & 67,53 & 67,53 & 67,53 \\
\hline Teor Fe Rejeito (\%) & 19,61 * & 11,92 & 9,73 \\
\hline Recuperação Mássica na Flotação (\%) & 65,45 & 70,23 & 71,35 \\
\hline Massa de produto da flotação (t) & 16.147 .567 & 17.326 .434 & 17.604 .771 \\
\hline Massa adicional de produção da flotação (t) & - & 1.178 .867 & 1.457 .204 \\
\hline Massa adicional de produção da flotação (\%) & - & 7,3 & 9,0 \\
\hline Recuperação mássica no Peneiramento (\%) & - & 91,7 & 89,0 \\
\hline Massa de produto do peneiramento $(t)$ & - & 1.081 .051 & 1.296 .834 \\
\hline Produção Total de Pellet Feed (t) & 16.147 .567 & 17.228 .618 & 17.444 .401 \\
\hline$(\Delta)$ Produção Total de Pellet Feed $(t)$ & - & 1.081 .051 & 1.296 .834 \\
\hline$(\Delta)$ Produção de Pellet Feed $(\%)$ & - & 6,7 & 8,0 \\
\hline \multirow{3}{*}{\multicolumn{2}{|c|}{$\begin{array}{l}\text { Observaçöes: } \\
\text { - Todos os valores relacionados acima são anuais e foram retirados do Balanço de } \\
\text { Massa ROM } 2014 \text { - Consolidado GAEHS. }\end{array}$}} & \multicolumn{2}{|c|}{ Preços de Insumos - Ref. Jan/2014 a Jun./14 } \\
\hline & & Custo $\mathrm{NaOH}$ (R\$/ton) & $\mathrm{R} \$ 4.000,00$ \\
\hline & & Custo amido (R\$/ton) & $\mathrm{R} \$ 800,00$ \\
\hline
\end{tabular}

* Contribuição técnica ao $44^{\circ}$ Seminário de Redução de Minério de Ferro e Matérias-primas, 15o Simpósio Brasileiro de Minério de Ferro e 2o Simpósio Brasileiro de Aglomeração de Minério de Ferro, 15 a 18 de setembro de 2014, Belo Horizonte, MG, Brasil. 
Os dados da tabela 12 mostram que a elevação do pH no circuito scavenger de flotação para valores acima de 10, seja através da adição de soda ou amido gelatinizado pode resultar em um aumento adicional e significativo de recuperação para a operação de flotação. Considerando que uma pequena parte desta massa adicional recuperada será descartada através do peneiramento de alta frequência em função do engrossamento do concentrado devido a presença de sílica grosseira, ainda sim os ganhos finais de produção justificam a implementação desta melhoria ao processo.

Quanto à utilização de soda ou amido gelatinizado para obtenção dos níveis de recuperação propostos, a opção pela segunda alternativa se mostra mais viável devido ao maior ganho de produção, embora se saiba que haverá um custo adicional de reagentes.

\section{CONCLUSÃO}

Os resultados obtidos mostram que é possível reduzir o teor de Fe no rejeito da flotação scavenger de Brucutu para próximo de 10\%, através da elevação do pH para valores acima 10,0 utilizando-se amido gelatinizado. A expectativa de um acréscimo na produção da usina da ordem de 1.300 .000 t/a é real (5,5\%), visto que a implementação desta melhoria é relativamente simples e os ganhos são bastante expressivos.

\section{REFERÊNCIAS}

1 Lima NP. (2001) Comportamento de minérios itabiríticos dos complexos Alegria e Fábrica Nova frente aos processos de deslamagem e flotação. Dissertação de Mestrado (Curso de Pós-Graduação em Engenharia Metalúrgica e de Minas). Escola de Engenharia da UFMG, Belo Horizonte, MG.

2 Peres AEC, Coelho EM, Araujo AC. (1980) Flotação, espessamento, deslamagem e floculação seletiva. In: Coelho EM. Tratamento de Minérios e Hidrometalurgia; In Memorian Prof. Paulo Abib Andery. Recife: ITEP-CNPq.

3 Lipper G. (2012) Adequação do depressor amido na flotação reversa de minério de ferro da usina de Brucutu - Contribuição técnica para o $40^{\circ}$ Seminário de Redução de Minério de Ferro e Matérias-Primas e $11^{\circ}$ Simpósio Brasileiro de Minério de Ferro.

4 Leja J. (1982) Surface Chemistry of Froth Flotation. New York: Plenum Press, 758p.

* Contribuição técnica ao 44 Seminário de Redução de Minério de Ferro e Matérias-primas, 15은 Simpósio Brasileiro de Minério de Ferro e $2^{\circ}$ Simpósio Brasileiro de Aglomeração de Minério de Ferro, 15 a 18 de setembro de 2014, Belo Horizonte, MG, Brasil. 